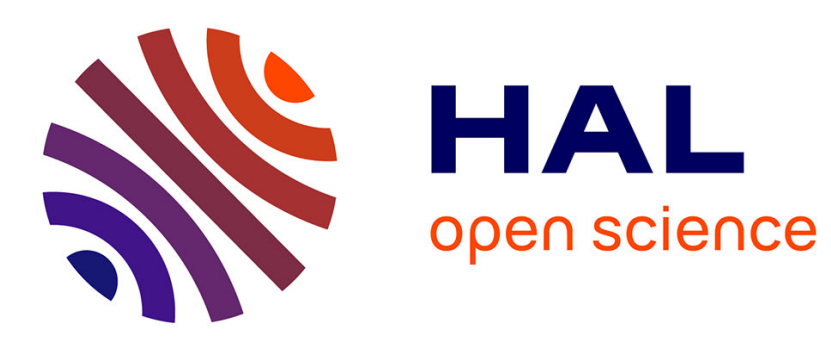

\title{
Kinetic simulations of ion temperature measurements from retarding field analyzers
}

\author{
F. Valsaque, G. Manfredi, J. P. Gunn, E. Gauthier
}

\section{To cite this version:}

F. Valsaque, G. Manfredi, J. P. Gunn, E. Gauthier. Kinetic simulations of ion temperature measurements from retarding field analyzers. Physics of Plasmas, 2002, 9 (5), pp.1806-1814. 10.1063/1.1463416. hal-03313004

\section{HAL Id: hal-03313004 \\ https://hal.science/hal-03313004}

Submitted on 3 Aug 2021

HAL is a multi-disciplinary open access archive for the deposit and dissemination of scientific research documents, whether they are published or not. The documents may come from teaching and research institutions in France or abroad, or from public or private research centers.
L'archive ouverte pluridisciplinaire HAL, est destinée au dépôt et à la diffusion de documents scientifiques de niveau recherche, publiés ou non, émanant des établissements d'enseignement et de recherche français ou étrangers, des laboratoires publics ou privés. 
N.B.

for editor version (or to cite this work) please refer to:

F. Valsaque, G. Manfredi, J. Gunn, E. Gauthier

"Kinetic simulations of ion temperature measurements from retarding field analyzers"

Physics of Plasmas, American Institute of Physics, 2002, 9 (5), pp.1806-1814.

DOI: $10.1063 / 1.1463416$

\title{
Kinetic simulations of \\ ion temperature measurements \\ from retarding field analyzers
}

\author{
F. VALSAQUE ${ }^{a)}$, G. MANFREDI \\ Laboratoire de Physique des Milieux Ionisés, Unité CNRS 7040, Université Henri Poincaré, \\ B.P. 239, 54506 Vandouvre - lès - Nancy, France
}

\section{J. P. GUNN, E. GAUTHIER}

Association CEA-EURATOM sur la fusion contrôlée, 13108 Saint Paul Lez Durance, France

\begin{abstract}
$\underline{\text { Abstract }}$
Retarding field analyzers (RFA) provide an integral of the ion velocity distribution in tokamak edge plasmas, leading, in principle, to an estimate of the ion temperature. However, the presence of the RFA itself perturbs the ambient plasma, such that the measured distribution is distorted with respect to the unperturbed one far from the probe. Here, collisionless kinetic modeling is employed to investigate the modification of the plasma characteristics (temperature, particle flux, density, and electric potential) in the presheath of the RFA. The kinetic equations are solved independently by means of two different numerical
\end{abstract}


methods, which provide a reliable check of their results. Moreover, they are interpreted in light of a simplified kinetic analytical model. Systematic numerical studies are performed for a large range of values of the ion-to-electron temperature ratio and the parallel drift speed. In the same way that a Mach probe measures upstream-downstream asymmetries of ion saturation current in flowing plasmas, RFAs are expected to measure important asymmetries of sheath potential and ion temperature. These asymmetries can be used to estimate accurately the ion temperature in the absence of the probe perturbation.

\section{Introduction}

A good knowledge and control of edge plasma conditions are necessary to maintain a steady state in fusion devices. In particular, material edge tokamak structures such as antennae, limiters, or divertor plates are eroded by the impact of energetic particles. Such a flux generates impurities by physical sputtering, which can be ionized in the scrape-off layer (SOL) and transported into the core plasma, thus degrading fusion energy and confinement ${ }^{1,2}$. In order to assess the incident power flux on material surfaces and the magnitude of impurity generation, the ion energy distribution and temperature are determining parameters. However, measuring accurately the latter is a difficult task. Spectroscopic measurements correspond to an average over a volume of plasma and do not provide directly the ion temperature, as hydrogen isotopes do not emit photons ${ }^{3}$. The ion temperature is estimated from the neutral temperature and an ion-neutral coupling model is therefore required ${ }^{4-6}$. An alternative technique, using a RFA device (retarding field analyzer) ${ }^{7-11}$ that measures directly the ion energy distribution, is investigated here by means of a kinetic model and numerical simulations.

\footnotetext{
a) corresponding author's email address: Fabrice.Valsaque@lpmi.uhp-nancy.fr
} 
A schematic description of the RFA is represented in Fig. 1. It consists of a small entrance slit in the probe surface, two grids and a collector. The probe is aligned along the magnetic field lines so as to measure the parallel component of the ion flux. The entrance slit is sufficiently biased to a negative (and constant) potential $\phi_{\mathrm{e}}$, so that most of the electrons coming from the plasma are repelled. The retarding potential $\phi_{\mathrm{S}}$ applied to the first grid ranges from zero to large positive values in order to scan the ion distribution function. Only the ions with a kinetic energy larger than $e \phi_{S}$ are collected. The second grid is negatively biased to a negative (constant) voltage $\phi_{\text {er }}$ to repel energetic electrons from the plasma (which pass the barrier potential of the entrance slit) and cancel out secondary electron emission created by ion impact on the collector. The entrance slit width is of the order of a Debye length or less ${ }^{11}$, so that it is shielded by the sheath. In this case, the ion distribution function entering the analyzer is reasonably close to the one reaching its external surface, and most incident electrons are repelled back into the plasma.

The experimental device RFA has already been employed in various domains of plasma physics $^{12-15}$, although the high heat flux released in the SOL and the smallness of the Debye length had limited its use for tokamak plasmas in the past ${ }^{7-10}$. Nowadays, even if this problem can be overcome by an appropriate design of the analyzer ${ }^{11,16}$, there are still some difficulties, particularly because RFA measurements strongly depend on the plasma flow. As large plasma flows are frequently observed in the $\mathrm{SOL}^{17-20}$, their effect should be taken into account to provide a realistic interpretation of the measurements. The latter point will be addressed in the present paper. 
Various types of models, both fluid and kinetic, have been used in the past for the theoretical and numerical analysis of the plasma-probe transition layer. As the ion density is depleted by the probe, the dynamical equations must be supplemented by an ad hoc source term, in order to reach an equilibrium state in the presheath. Different ion sources were proposed by Emmert et $a .^{21}$ and Bissel and Johnson ${ }^{22}$, but, as their studies were restricted to the case of a plasma bounded between two walls, no plasma flow was considered. Stangeby ${ }^{23}$ proposed a fluid model suitable for the case of tokamak edge plasmas, but the source he used only permitted cross-field diffusion from the plasma into the presheath, and not vice versa. Even though in strong magnetic field situations, the inward (plasma to presheath) transport is larger than the outward transport, the latter cannot be neglected. A more realistic source, accounting for ion exchange in both directions, was introduced by Hutchinson ${ }^{24}$. This modification significantly improved the realism of the model, which has since shown to be in good agreement with experimental results ${ }^{25-26}$. However, the fluid approach adopted in Ref. 24 assumed isothermal ions, which is not entirely satisfactory in such plasma-probe transition layers. In a subsequent paper, Chung and Hutchinson ${ }^{27}$ generalized this approach by using a kinetic model, which provides direct information on the ion distribution and is no more restricted by the isothermal assumption. For the sake of the interpretation of ion temperature measurements by RFA, the kinetic approach is crucial as the shape of the ion distribution function (which is rarely Maxwellian) plays a considerable role.

The kinetic model developed by Chung and Hutchinson ${ }^{27}$, in the context of Mach probes ${ }^{16}$, is applied to the case of RFA in Sect. II. Section III starts with a quick description of the two different numerical approaches to solve the model's equations. Then, Sect. III contains our interpretations of the numerical results and their comparison with analytical solutions derived from a simplified kinetic model. We discuss the asymmetries between RFA measurements 
taken on each side of the probe. Furthermore, the plasma drift and plasma ion temperature effects are studied. Conclusions and discussions on the experimental applications are reported in Sect. IV.

\section{Model: probe in a strong magnetic field}

\section{A. Governing equations}

In order to model the plasma-probe interaction in the SOL of tokamak plasmas, we consider the interaction of a collisionless flowing plasma with a fixed wall, in the presence of a strong uniform magnetic field, following the approach of Chung and Hutchinson ${ }^{27}$. The density perturbation caused by the probe can be characterized by two regions: an electrically charged Debye sheath and a quasineutral presheath. The Debye sheath is found in the immediate vicinity of the wall and is a few Debye lengths thick. In typical tokamak edge plasmas, the Debye sheath thickness is of the order of $0.1 \mathrm{~mm}^{28}$, whereas the diameter of the probe tends to be a few centimeters. On a macroscopic scale the Debye sheath is negligible; it is a thin, collisionless, and sourceless transition layer that serves to balance the ion and electron fluxes to the probe surface. The quasineutral presheath region extends along the field lines inside the flux tube connected to the probe. The presheath length is determined by the balance between the parallel flow normal to the probe surface and the cross-field transport that feeds the presheath from the unperturbed plasma outside the flux tube (Fig. 2). The probe considered is, indeed, a double-mounted RFA, which can provide simultaneous measurements from both sides. The magnetic field is strong enough that the ion gyroradius is significantly smaller than the size of the probe. In most tokamak SOLs with magnetic field strengths of a few Teslas and ion temperatures some tens of electron volts, the Larmor radius is typically a few tenths of a 
millimeter. In this case, the cross-field transport can be considered as being diffusive (generally anomalous $)^{27}$ and is modeled as a random migration of ions across magnetic field lines. The migration rate is governed by the magnetic field strength, so that, for typical SOL regimes, parallel convection dominates over perpendicular transport. Therefore, the parallel length of the presheath $L_{/ /}$is very long compared to the cross-field dimension of the probe $L \perp$. For instance, typical values for the Tore Supra tokamak are $L_{/ /}=20 \mathrm{~m}$ and $L_{\perp}=2 \mathrm{~cm}$ with $c_{\mathrm{S}}=50 \mathrm{~km} / \mathrm{s}$ and $D_{\perp}=1 \mathrm{~m}^{2} \mathrm{~s}^{-1}$, where $c_{\mathrm{S}}$ and $D_{\perp}$ are respectively the acoustic velocity and the cross-field diffusion coefficient. In this case, we can use a one-dimensional model in the parallel direction, the cross-field transport being taken into account by a simplified source term $^{27,29}$. For a collisionless presheath, the ion dynamics is governed by the Vlasov equation. Its parallel projection in case of singly charged ions can be written as:

$\frac{\partial f_{\mathrm{i}}}{\partial t}+v \frac{\partial f_{\mathrm{i}}}{\partial x}-\frac{\partial \phi}{\partial x} \frac{e}{m_{\mathrm{i}}} \frac{\partial f_{\mathrm{i}}}{\partial v}=S$

where $f_{\mathrm{i}}(x, v, t)$ is the ion distribution function in the presheath, $v$ the ion parallel velocity, $e$ the ion charge, $\phi(x, t)$ the presheath electric potential computed self-consistently from the ion distribution, $m_{\mathrm{i}}$ the ion mass and $S$ the volume source term. As the latter corresponds to a random migration of ions across magnetic field lines, exchanges between the unperturbed plasma and the presheath must be considered. We assume that these exchanges occur, in both directions, at a frequency $W=D_{\perp} / L_{\perp}{ }^{2}$. The volume source $S$ therefore becomes:

$S=W\left(f_{0}-f_{\mathrm{i}}\right)$ 
where $f_{0}(v)$ is the ion distribution function in the unperturbed plasma. The first term on the right hand side of Eq. (2) models ions entering the presheath from the unperturbed plasma and the second ions exchanged in the other direction. Assuming electrons at thermal equilibrium, their density in the presheath is given by the Boltzmann relation:

$n_{\mathrm{e}}(x, t)=n_{0} \exp \left(e \phi(x, t) / k_{\mathrm{B}} T_{\mathrm{e}}\right)$

where $n_{0}$ is the unperturbed plasma density, $k_{\mathrm{B}}$ is Boltzmann's constant and $T_{\mathrm{e}}$ the electron temperature.

This set of equations is closed by the quasineutrality condition:

$$
\frac{e \phi(x, t)}{k_{\mathrm{B}} T_{\mathrm{e}}}=\ln \left(\frac{\int f_{\mathrm{i}} d v}{n_{0}}\right)
$$

which implicitly assumes that $\lambda_{\mathrm{De}}=0$, so that the Debye sheath is neglected.

\section{B. Normalized equations}

Assuming quasineutrality and a constant ion exchange frequency $W$, the previous selfconsistent equations are normalized with the following transformations:

$$
t^{\prime}=W t, \quad v^{\prime}=\frac{v}{c_{\mathrm{e}}}, \quad x^{\prime}=\frac{W x}{c_{\mathrm{e}}} \quad \text { and } \quad \phi^{\prime}=\frac{e \phi}{k_{\mathrm{B}} \mathrm{T}_{\mathrm{e}}}
$$


where we define the cold ion sound speed $c_{\mathrm{e}}=\left(k_{\mathrm{B}} T_{\mathrm{e}} / m_{\mathrm{i}}\right)^{1 / 2}$ as a type of acoustic velocity. In our treatment, we do not use a fluid approximation, such as the assumption of isothermal or adiabatic behavior for the ions, so that the choice of a precise definition of the acoustic velocity is not important. This normalization, which is useful to express velocities with an appropriate unit, will be used throughout the rest of this paper. By dropping the primes for brevity on the quantities defined in Eq. (5), Eqs. (1-4) can thus be written in the form:

$$
\begin{aligned}
& \frac{\partial f_{\mathrm{i}}}{\partial t}+v \frac{\partial f_{\mathrm{i}}}{\partial x}-\frac{\partial \phi}{\partial x} \frac{\partial f_{\mathrm{i}}}{\partial v}=f_{0}-f_{\mathrm{i}} \\
& \phi(x, t)=\ln \int f_{\mathrm{i}} d v
\end{aligned}
$$

Outside the presheath, the ion distribution function $f_{0}$ is assumed to be a shifted Maxwellian with temperature $\mathrm{T}_{\mathrm{i} 0}$ and mean velocity $U_{0}$. The expression of $f_{0}$ is thus

$$
f_{0}=\frac{1}{\sqrt{2 \pi \tau}} \exp \left(-\frac{\left(v-U_{0}\right)^{2}}{2 \tau}\right)
$$

where $\tau=T_{\mathrm{i} 0} / T_{\mathrm{e}}$.

We assume that the probe surface (located at $x=0$ ) is perfectly absorbing, and that far from the probe the ion distribution is equal to $f_{0}$. Besides, using Eqs. (7-8), the boundary conditions become:

$$
f_{\mathrm{i}}(x=0, v>0)=0, \quad f_{\mathrm{i}}(x \rightarrow \infty)=f_{0}, \quad \phi(x \rightarrow \infty)=0
$$


on the upstream side and

$f_{\mathrm{i}}(x=0, v<0)=0, f_{\mathrm{i}}(x \rightarrow-\infty)=f_{0}, \quad \phi(x \rightarrow-\infty)=0$

on the downstream side (see Fig. 2).

Therefore, considering this self-consistent set of equations, the presheath behavior is governed by only two dimensionless parameters of the unperturbed plasma, which are the ion to electron temperature ratio $\tau$ and the mean ion velocity $U_{0}$ (normalized to $c_{\mathrm{e}}$ ) also called the plasma drift velocity. The ion particle density, current density, mean speed, and kinetic temperature are computed as moments of $f_{\mathrm{i}}$ :

$n_{\mathrm{i}} \equiv \int f_{\mathrm{i}} d v \quad J_{\mathrm{i}} \equiv \int u f_{\mathrm{i}} d v \quad U \equiv \frac{J_{\mathrm{i}}}{n_{\mathrm{i}}} \quad T_{\mathrm{i}, \mathrm{kin}} \equiv \frac{1}{n_{\mathrm{i}}} \int(v-U)^{2} f_{\mathrm{i}} d v$

\section{Results}

Numerical results are always generated with an inaccuracy inherent to the method used. We solved independently the set of Equations (6-7), with the boundary conditions Eqs. (9-10), by means of two different numerical approaches. The results are obtained by initializing the codes with a spatially uniform ion distribution, which is then left to evolve self-consistently until it reaches a stationary equilibrium. The first numerical tool is a rendered-down version of the two-dimensional GUNDY particle-in-cell (PIC) ${ }^{30}$ code used to simulate ion flows around a cylindrical Gundestrup probe ${ }^{26}$. The presheath is adequately described by 100 cells containing 2000-4000 particles each. The second numerical tool is a Vlasov-Eulerian code ${ }^{31-}$ 34. It computes directly the ion distribution function on a grid corresponding to the phase space variables $(x, v)$, which are both sampled with $200-400$ points. As it will be presented in 
the following, results from both numerical approaches are in good agreement in all the cases studied. We interpret this agreement as an indication that numerical inaccuracy does not significantly affect the results.

\section{A. Ion distributions and probe characteristics for $\tau=2$ and $U_{0}=1$}

The primary goal of this paper is to propose a method to extract the ion temperature in the unperturbed plasma from quantities measured by the RFA. For the purpose of illustration, the two parameters controlling the presheath behavior are firstly set to $\tau=2$ and $U_{0}=1$; the ions are twice as hot as electrons and drifting with a parallel speed equal to $c_{\mathrm{e}}$ in the unperturbed plasma. The latter value corresponds to a fluid Mach number (drift velocity normalized to the acoustic velocity with isothermal ions $\left.c_{\mathrm{S}}=\left(k_{\mathrm{B}}\left(T_{\mathrm{e}}+T_{\mathrm{i}}\right) / m_{\mathrm{i}}\right)^{1 / 2}\right)$ of around 0.6 , which is consistent with expectations in SOL plasmas ${ }^{17-20}$. Referring to Figs. 1-2, ions moving towards the analyzer on the downstream side have positive speeds and vice versa on the upstream side. Figs. 3.a-b present the ion distribution function $f_{\mathrm{i}}(x, v)$ obtained from the Vlasov-Eulerian simulations, on both sides of the analyzer, at different positions. It shows the progressive modifications from the Maxwellian distribution at the plasma boundary to the one at the wall. (Strictly speaking, we calculate the distribution function at the sheath edge, but for brevity we refer to it here as the "wall". The actual distribution at the solid surface can be easily obtained by applying a shift in energy equal to the sheath potential drop.) On both sides, the ion density decreases, near the probe, as the integral of the velocity distribution becomes smaller. This depletion of the distribution function is due to the total absorption condition at the wall. On the upstream side (Fig. 3.b), the kinetic modifications concern almost only ions entering the presheath with a velocity not directed to the probe. The others are collected without being significantly accelerated, which indicates that the electric field is weak. The wall distribution 
is more distorted on the downstream side (Fig. 3.a) because of the heavy depletion by the probe of ions coming from the upstream direction. Therefore, the density is very low and the potential drop is large, as given by the Boltzmann relation [Eq. (7)]. Ions are then accelerated by the electric field in the direction of the wall. This shifts the distribution profiles towards positive values of the velocity. Further, as it was also noticed in a preliminary study ${ }^{35}$, the shape of the distribution is no longer Maxwellian (e.g. at $x=0.1$ the profile is clearly asymmetric), which points out that a kinetic model is indeed necessary for this kind of problem.

An analytical solution can be derived from the Vlasov equation, by neglecting the electric field. At equilibrium $\left(\partial f_{\mathrm{i}} / \partial t=0\right)$, Eq. (6) becomes:

$$
v \frac{\partial f_{\mathrm{i}}}{\partial x}=f_{0}-f_{\mathrm{i}}
$$

According to the boundary conditions specified in Eqs. (9-10), a solution for $f_{\mathrm{i}}$ is:

$$
f_{\mathrm{a}}(x, v)=f_{0}(v)\left(1-H( \pm v) \exp \left(-\frac{x}{v}\right)\right),
$$

where positive and negative signs stand respectively for upstream and downstream cases, and $H$ is the Heaviside function. It is useful to contrast this model with the full kinetic results in order to distinguish the importance of simple geometrical shadowing with respect to distortion of the distribution function by the electric field. The distribution profiles $f_{\mathrm{a}}$ at the same positions as those presented from the numerical results are plotted in Figs. 3.c-d. For velocities directed towards the analyzer (i.e. $v>0$ on the downstream side, and $v<0$ on the 
upstream side), all profiles are identical to the equilibrium Maxwellian distribution, because the ions are not accelerated. The function $f_{\mathrm{a}}$ is continuous even at the apparent singularity $v=0$, except for $x=0$ : as $x$ decreases, its gradient at $v=0$ becomes gradually steeper, until a discontinuity occurs. On the analyzer $(x=0)$, the analytical model yields a shifted Maxwellian, truncated at $v=0$. In the general case (for $U_{0} \neq 0$ ), the ion distributions are, thus, not simply half-Maxwellian. The shadowing effect of the probe yields a density decrease and thus a potential drop, which are both larger on the downstream side. Therefore, the electric field magnitudes on each side of the probe are not the same. This fact determines the relative degree of distortion of the distribution functions computed numerically and explains why the upstream analytical profiles are closer to the numerical results.

Fig. 4 shows, on both sides of the probe, the electrostatic potential, ion current, and ion kinetic temperature, which are computed from Eq. (11). Table I summarizes the values of such quantities, at the wall, for the different methods. Both numerical methods, PIC and VlasovEulerian, are in good agreement, especially for the profile variations. On the downstream side, as it was mentioned before, the density perturbation is larger, so that the wall potential is more negative. In the vicinity of the wall, the potential and kinetic temperature gradients are rather steep, which required the use of a non-uniform mesh to obtain accurate results. The kinetic temperature quantifies the narrowing, near the wall, of the distribution functions of Figs. 3.ab. However, this does not give any information on the distortions of such distributions. By using the same procedure for $f_{\mathrm{a}}$ as for $f_{\mathrm{i}}$ in Eq. (11), the analytical model gives the density, current, and temperature, which are also plotted in Fig. 4. Both analytical and numerical results present a substantial kinetic temperature drop near the analyzer, which is similar to the case of a presheath surrounding a Mach probe ${ }^{26}$. Here, an isothermal ion assumption is clearly not valid. Considering the drastic assumption made on the electric field, the analytical profiles 
are rather close to those obtained by solving numerically the full Vlasov equation. This points out that (for this case $\tau=2$ and $U_{0}=1$ ) the electric field effects are not predominant : the presheath behavior is mainly governed by the probe shadowing effect.

Table I. Density, electric potential, ion current and ion kinetic temperature, at the wall. (Quantities respectively normalized to $n_{0}, k_{\mathrm{B}} T_{\mathrm{e}}, n_{0} c_{\mathrm{e}}$ and $T_{\mathrm{e}} ; \tau=2$ and $U_{0}=1$.)

\begin{tabular}{lcccccccc} 
& \multicolumn{3}{c}{ Downstream } & & \multicolumn{3}{c}{ Upstream } \\
& $n_{\mathrm{i}}$ & $\phi$ & $J_{\mathrm{i}}$ & $T_{\mathrm{i}, \mathrm{kin}}$ & $n_{\mathrm{i}}$ & $\phi$ & $J_{\mathrm{i}}$ & $T_{\mathrm{i}, \mathrm{kin}}$ \\
& & & & & & & & \\
& 0.21 & -1.56 & 0.31 & 0.43 & 0.67 & -0.40 & -1.25 & 0.94 \\
\hline PIC & 0.19 & -1.66 & 0.31 & 0.37 & 0.66 & -0.42 & -1.25 & 0.91 \\
\hline Vlasov & & & & & & & & \\
\hline Analytical & 0.24 & -1.43 & 0.20 & 0.47 & 0.76 & -0.27 & -1.20 & 1.09 \\
& & & & & & & & \\
\hline \hline
\end{tabular}

Concerning the measurements by the RFA (see Fig. 1), the retarding potential $\phi_{s}$ applied to the ion selector affects only the ions whose kinetic energy is too small to reach the collector. Therefore, the differential fluxes at the wall $v f_{\mathrm{i}}(x=0, v)$, can be scanned by varying $\phi_{\mathrm{S}}$ on each side. Provided that the potential on the collector is lower than the one at the entrance slit $\left(\phi_{\mathrm{c}}<\phi_{\mathrm{e}}\right)$, all the ions passing through the ion selector reach the collector (even for $\phi_{\mathrm{S}}=0$ ). The upstream and downstream collected currents are then:

$$
J_{\mathrm{C}, \text { Down }}=\int_{\sqrt{2 \phi_{S}}}^{\infty} v f_{\mathrm{i}}(x=0, v) d v \quad J_{\mathrm{C}, \mathrm{U} p}=\int_{-\infty}^{-\sqrt{2 \phi_{S}}} v f_{\mathrm{i}}(x=0, v) d v
$$

Collected currents vary from their maximum values (obtained for $\phi_{S}=0$ ) to zero (for large positive values of $\phi \mathrm{s})$. RFA characteristics, which are the semi-logarithm plots presented in 
Fig. 5, are the kind of results obtained experimentally. In our computations, the Debye sheath was neglected, so the numerical results correspond only to the decreasing part of the curve. However, the plateau region in Fig. 5 can be explained as follows. The entrance slits (see also Fig. 1), on both side of the RFA, are usually negatively biased to the same value $\phi_{\mathrm{e}}$ (lower than $\left.\phi_{\mathrm{ps}}=\phi(x=0)\right)$. Before entering the RFA, all ions are accelerated by the potential drop $\phi_{\mathrm{d}} \equiv \phi_{\mathrm{ps}}-\phi_{\mathrm{e}}$, which is considered occurring within the collisionless Debye sheath. Therefore, as long as $\phi_{\mathrm{S}}$ is lower than $\phi_{\mathrm{d}}$, no ions are deflected and the collected current remains maximal. The electric potential $\phi_{\mathrm{ps}}$ at the wall $(x=0)$, is larger (in absolute value) on the downstream side, therefore the potential drop $\phi_{\mathrm{d}}$ is smaller. The absolute value of $\phi_{\mathrm{e}}$ does not really matter, as it just shifts the two characteristics by the same voltage. What is more important is the relative difference between them, which is $\Delta \phi \equiv \phi_{\mathrm{d}, \text { up }}-\phi_{\mathrm{d}, \text { down }}=\phi_{\text {up }}(x=0)-$ $\phi_{\text {down }}(x=0)$ : we call this quantity upstream-to-downstream potential difference.

An estimated ion temperature $T_{\mathrm{RFA}}=-1 / \alpha$ can be deduced from the slope $\alpha$ of the linear part of the characteristics ${ }^{11}$. We computed the slopes by using a least square method. This estimation would give the correct ion temperature only if the ion distribution on the wall were a half-Maxwellian with no shift, which is clearly not the case, as shown in Fig. 3. The kinetic modifications can be significant, so it is not surprising that the measured value is not equal to the equilibrium plasma temperature $T_{\mathrm{i} 0}$. However, by means of measures on both sides of the analyzer, as we will explain, a more accurate estimation can be obtained. As most characteristics are not linear on their upper part and very small currents are not experimentally measurable, we need to restrict the range of data to attempt to simulate what one can really measure. On the other hand, such range must be sufficiently wide to have enough data to compute accurately the slope. We have used a standard procedure, which corresponds to a good compromise for all values of $\tau$ and $U_{0}$, by fitting the numerical values for which the 
logarithm of the current is included between $\ln \left(\left|J_{\max }\right|\right)-1$ and $\ln \left(\left|J_{\max }\right|\right)-4$, where $J_{\max }$ is the current collected for $\phi_{\mathrm{S}}=0$. The analytical model gives, by using $f_{\mathrm{a}}$ in Eq. (14):

$\left|J_{\mathrm{C}, \mathrm{a}}\right|=\frac{U_{0}}{2}\left\{\operatorname{erf}\left(\frac{U_{0}}{\sqrt{2 \tau}} \mp \sqrt{\frac{\phi_{\mathrm{S}}}{\tau}}\right) \pm 1\right\}+\sqrt{\frac{\tau}{2 \pi}} \exp \left(-\frac{\left(\sqrt{2 \phi_{\mathrm{S}}} \mp U_{0}\right)^{2}}{2 \tau}\right)$

where upper and lower signs refer respectively to upstream and downstream cases and erf is the error function. In Fig. 5, both the analytical and the numerical characteristics are shifted by the constant value $\phi_{\mathrm{d}}$. On the downstream side, the analytical curve does not fit well the numerical data. However, we are only interested in the slope of its linear part. The RFA temperature as a function of the retarding potential $\phi_{\mathrm{S}}$ can be defined as the inverse of the local slope of the curve:

$T_{\mathrm{RFA}}\left(\phi_{\mathrm{S}}\right) \equiv-\left(\frac{\partial \ln \left|J_{\mathrm{C}}\right|}{\partial \phi_{\mathrm{S}}}\right)^{-1}$

The analytical model gives:

$T_{\mathrm{RFA}, \mathrm{a}}\left(\phi_{\mathrm{S}}\right)=\tau \pm U_{0} \sqrt{\frac{\pi \tau}{2}}\left\{\operatorname{erf}\left(\frac{ \pm U_{0}}{\sqrt{2 \tau}}-\sqrt{\frac{\phi_{\mathrm{S}}}{\tau}}\right)+1\right\} \exp \left(\frac{\left(\sqrt{2 \phi_{\mathrm{S}}} \mp U_{0}\right)^{2}}{2 \tau}\right)$

The first term in Eq. (17) corresponds to the result obtained by Pitts ${ }^{11}$ with a non-shifted halfMaxwellian distribution and coincides with the temperature in the plasma core. The second term is the correction due to the drift of the plasma and vanishes for $U_{0}=0$. It is positive on the upstream side and negative on the downstream side. Therefore, one can expect that the 
measured RFA temperature is larger than the real ion temperature on the upstream side, and lower on the downstream side. As $\phi_{\mathrm{S}}$ increases, the error function in Eq. (17) tends to -1 faster than the exponential function grows. Thus, the analytical RFA temperature remains finite and tends to $\tau$ as $\phi_{\mathrm{S}}$ becomes larger (an asymptotic expansion to the first order gives $\left.T_{\mathrm{RFA}, \mathrm{a}}\left(\phi_{\mathrm{S}} \rightarrow \infty\right) \propto \tau \pm U_{0} \tau /\left(2 \phi_{\mathrm{S}}\right)^{1 / 2}\right)$. However, since for large values of the potential the current is rather small, this limit is not interesting from an experimental point of view. A Taylor series of Eq. (17) with respect to the variable $U_{0}$, shows that odd terms in the expansion for the downstream case are opposite in sign to the odd terms for the upstream case. Thus, the average RFA temperature:

$$
\bar{T}_{\mathrm{RFA}} \equiv \frac{T_{\mathrm{RFA}, \mathrm{up}}+T_{\mathrm{RFA}, \mathrm{down}}}{2}
$$

is equal to $\tau$ to the second order in the expansion. Therefore, even if the RFA temperature on each side of the analyzer is not equal to the one in the plasma, we can expect that the estimation from Eq. (18) gives accurate results as long as the plasma drift is not too important.

For the sake of comparison with the numerical results, we estimated the analytical RFA temperature within the same current interval, i.e. $\ln \left(\left|J_{\max }\right|\right)-1>\ln J_{c, \text { a }}>\ln \left(\left|J_{\max }\right|\right)-4$. As $\mathrm{T}_{\mathrm{RFA}, \mathrm{a}}$ does not depend on $J_{\mathrm{c}, \mathrm{a}}$ but rather on $\phi_{\mathrm{S}}$, the corresponding retarding potential interval $\left(\phi_{\mathrm{S} 1}<\phi_{\mathrm{S}}<\phi_{\mathrm{S} 4}\right)$, for each set of values of $\tau$ and $U_{0}$, is found numerically by means of a dichotomy method. Then $\mathrm{T}_{\mathrm{RFA}, \mathrm{a}}$ is averaged between $\phi_{\mathrm{S} 1}$ and $\phi_{\mathrm{S} 4}$ :

$$
T_{\mathrm{RFA}, \mathrm{a}}=\frac{1}{\phi_{\mathrm{S} 4}-\phi_{\mathrm{S} 1}} \int_{\phi_{\mathrm{S} 1}}^{\phi_{\mathrm{S} 4}} T_{\mathrm{RFA}, \mathrm{a}}\left(\phi_{\mathrm{S}}\right) d \phi_{\mathrm{S}} .
$$


Table II gives the comparison between RFA temperatures obtained by different methods. As the RFA temperature is estimated within a current range, the scan of the ion distribution function is restricted within an energetic range (too low or too high energy ions in the distribution are not taken into account). The RFA temperature, then, corresponds to the temperature of a Maxwellian distribution that best fits the ion distribution within this energetic range. The ion distributions analyzed are not Maxwellian, so that this is not a measure of the standard deviation as given by the kinetic temperature, which explains why the RFA temperatures are different from those shown in Table I. Average RFA temperatures are equal to $\tau$ (the plasma temperature) with a relative error lower than $3 \%$.

Table II. Downstream, upstream and average RFA temperatures (normalized to $T_{\mathrm{e}} ; \tau=2$ and $\left.U_{0}=1\right)$.

\begin{tabular}{lccc}
\hline \hline$\tau=2, U_{0}=1$ & $T_{\text {RFA,down }}$ & $T_{\text {RFA,up }}$ & $\bar{T}_{\text {RFA }}$ \\
\hline PIC & 1.52 & 2.58 & 2.05 \\
Vlasov & 1.51 & 2.58 & 2.05 \\
Analytical & 1.50 & 2.62 & 2.06 \\
\hline \hline
\end{tabular}

\section{B. Drift and ion temperature effects}

Keeping the procedure presented in Sect. III.A, we now investigate a larger range of the plasma parameters: $\tau$ varying from 0.1 to 5 , and $U_{0}$ varying from zero to $c_{\mathrm{S}}$, where $c_{\mathrm{S}}$ is the acoustic velocity with isothermal ions $c_{\mathrm{S}}=c_{\mathrm{e}}(1+\tau)^{1 / 2}$. 
The agreement between PIC and Vlasov-Eulerian results was verified for each set of values $\left(\tau, U_{0}\right)$. The physical correctness of the results was checked in two ways. Firstly, a few runs with zero electric field were performed to compare with the analytic solution, and excellent agreement was found. Secondly, fluid moments of the ion distribution provide a simple test to confirm the validity of the numerical results. Integration of the Vlasov equation (6), with respect to $v$, and using the definitions of Eq. (11), gives at equilibrium $\left(\partial f_{\mathrm{i}} / \partial t=0\right)$ :

$$
\frac{\partial J_{\mathrm{i}}}{\partial x}=1-n_{\mathrm{i}}
$$

Typically, in our simulations, Eq. (20) is verified with an error below $0.4 \%$.

In Fig. 6, the ratio of upstream-to-downstream collected current (for $\phi_{S}=0$ ) is plotted versus the Mach number (equal to the drift velocity normalized to the acoustic velocity $c_{\mathrm{S}}$ ) and for different values of the parameter $\tau$ (from 0.1 to 5 ). As both numerical methods give very similar results, only those from the Vlasov-Eulerian computations are presented. For each $\tau$, the current ratio curves are remarkably straight on a semi-logarithm scale, as it was pointed out by Chung and Hutchinson ${ }^{27}$. By expressing the plasma drift speed in units of $c_{\mathrm{S}}$ instead of $c_{\mathrm{e}}$, all current ratio curves have almost the same slope. Therefore, this kind of plot can be used to calibrate Mach probes ${ }^{26,36}$, as the value of $\tau$ is not required to estimate the dimensionless Mach number. The upstream-to-downstream RFA temperature ratio and upstream-todownstream difference potential $\Delta \phi$, plotted versus the plasma drift speed $U_{0}$, also yield relatively straight lines (see Fig. 7) for $\tau \geq 1$. It appears that both $\Delta \phi$ and the RFA temperature ratio increase with $U_{0}$ and decrease with $\tau$. The dependence on $U_{0}$ can be expressed as: 


$$
\frac{J_{\mathrm{C}, \text { up }}(\phi=0)}{J_{\mathrm{C} \text {,down }}(\phi=0)}=\exp \left(K_{\mathrm{J}}(\tau) U_{0}\right), \quad \frac{T_{\mathrm{RFA}, \text { up }}}{T_{\mathrm{RFA} \text {, down }}}=\exp \left(K_{\mathrm{T}}(\tau) U_{0}\right), \quad \Delta \phi=K_{\phi}(\tau) U_{0},
$$

where $K_{\mathrm{J}}(\tau), K_{\mathrm{T}}(\tau)$ and $K_{\phi}(\tau)$ are computed with a least-square method to fit the curves of Figs. 6 and 7. The dependence of such quantities on $\tau$ is shown in Fig. 8.

For cold ions $(\tau=0.1$ or 0.2$)$, the semi-logarithm plots of RFA temperature ratio versus the plasma drift speed $U_{0}$ are not linear. For these values of $\tau$, the second equality of Eq. (21) is not a good approximation. This fact is illustrated in Fig. 9, which shows the numerical and analytical RFA temperatures [computed from Eq. (16)] versus the retarding potential, on the downstream case for $U_{0}=0.5$ and $\tau$ ranging from 0.1 to 2 . As it was mentioned in Sect. III.A: (i) a non-shifted Maxwellian distribution $\left(U_{0}=0\right)$ would give [see Eq. (17)] $T_{\mathrm{RFA}}(\phi \mathrm{s})=\tau$ $\forall \phi_{\mathrm{S}}$; (ii) by taking into account the shift, downstream RFA temperatures are lower than the corresponding values of $\tau$; (iii) RFA characteristics are not linear on their upper part (see Fig. 5), and therefore $T_{\mathrm{RFA}}\left(\phi_{\mathrm{S}}\right)$ is not a significant quantity for small values of the retarding potential. Figure 9 shows that for cold ions it is less justified to deduce one single value of the RFA temperature from the RFA characteristic, as the latter is constant only in a narrow interval of the potential $\phi_{S}$. Since the analytical model neglects the presheath electric field, leading to a truncated Maxwellian distribution on the analyzer [see Eq. (13)], one can see qualitatively the differences due to the electric field on the kinetic distribution. For $\tau<1$ and as $\tau$ decreases, electric effects become larger, and the numerical curves of Fig. 9 are farther from the analytical ones. Therefore, deducing the plasma temperature from the RFA one is less accurate since the correlation between the ion distributions at the plasma boundary and on the analyzer is less clear. Moreover, for each value of $\tau$, the RFA temperature was estimated 
within a current interval compatible with the restrictions presented in Sect. III.A. These intervals are delimited by the thick vertical segments on each numerical curve of Fig. 9. One can see that they are less and less appropriate as $\tau$ decreases, since they do not correspond to the intervals where $T_{\mathrm{RFA}}\left(\phi_{\mathrm{S}}\right)$ is constant.

From Eq. (18), the average RFA temperature $\bar{T}_{\mathrm{RFA}}$ is plotted, in Fig. 10, versus the plasma drift speed $U_{0}$. According to Sect. III.A., the average temperature computed from the analytical model gives a good estimation of the ion plasma temperature $\tau$ as long as $U_{0}$ is not too large (typically $\left.U_{0} \leq 1\right)$. Except for cold ion plasmas $(\tau<1)$, the average RFA temperature obtained from the simulations remains close to $\tau$ even for greater drift velocities; for $\tau=1,2$, 3.5 and 5 , the relative differences between $\bar{T}_{\mathrm{RFA}}$ and $\tau$, are below $6,3,1.5$ and $1 \%$ respectively. For lower values of $\tau$, due to the problems described above, the ion temperature estimation is less accurate, although it remains roughly correct. In contrast, temperature estimations obtained from a single measure on either side of the RFA are far less accurate (see, for example, Table II). Therefore, the averaging procedure of Eq. (18) constitutes a reliable technique to deduce the ion temperature from RFA measurements.

\section{Conclusion}

The behavior of the presheath surrounding a RFA analyzer has been studied by means of theoretical analysis and numerical simulations of a kinetic model. The model incorporates the effect of self-consistent electric fields, global plasma drifts, as well as cross-field diffusion across magnetic field lines, and was first proposed by Chung and Hutchinson ${ }^{27}$ in a seminal paper. We have used two different numerical methods, particle-in-cell and Vlasov-Eulerian, which provides a reliable check for the results of the simulations. In addition, a simplified 
analytical model has been derived, by neglecting the effect of the electric field in the presheath.

In a tokamak environment, the ambient plasma far from the probe is characterized by a shifted Maxwellian distribution, with a well-defined temperature and average drift velocity. In the vicinity of the probe, the plasma is perturbed (mainly due to the shadowing effect induced by the probe itself), and its velocity distribution is no longer Maxwellian. The perturbation also depletes the ion density, and thus generates a self-consistent electric field. Our simulations have shown clearly how the equilibrium Maxwellian gets distorted in the presheath, both on the upstream side (where velocities are mainly directed towards the probe) and on the downstream side (where velocities are mainly directed opposite to the probe). The two sides of the probe are not symmetric. Modifications of the ion distribution function are more significant on the downstream side than on the upstream side, and consequently the density and electric potential drop are larger on the former than on the latter. Both PIC and VlasovEulerian codes yielded very similar results for the fully self-consistent problem. Less obviously, the results from the analytical model (which neglects the electric field) were also in relatively good agreement with the simulations, except for very low ion-to-electron temperature ratios. (This is not a severe limitation for measurements in SOL plasmas, as the ion temperature is generally believed to be close to or greater than that of the electrons ${ }^{1,6,10}$.) It appears therefore that the electric field plays only a secondary role in determining the structure of the presheath, at least for warm-ion regimes relevant to SOL plasmas. The primary effect in shaping the presheath originates from the geometric shadowing induced by the very presence of the probe.

The main purpose of the RFA is to measure the ion temperature. In experiments, this is done by plotting the current-voltage characteristics on a logarithmic scale: the ion temperature is 
then proportional to the inverse of the slope of this curve. This procedure, however, fails to incorporate the effect of the presheath on the ion velocity distribution, and yields a value of the ion temperature that is different from the "real" value in the unperturbed plasma. The difference is particularly significant when the plasma flow is large, as we observed in our simulations. Further, the results are different on each side of the probe: the measured temperature on the upstream side is larger than that on the downstream side. Nevertheless, we showed that an accurate estimation can be obtained by taking the average of the temperatures measured on each side of the probe. For ion temperatures relevant to tokamak SOL plasmas (i.e. $T_{\mathrm{i}} \geq T_{\mathrm{e}}$ ), this averaging procedure yields the unperturbed ion temperature within an accuracy of a few percent. For lower ion temperatures, the method is less accurate, although it still yields much better results than a single measure on either side of the analyzer.

Finally, other quantities than the ion temperature can be measured with RFAs. In particular, the analyzer can act as Mach probe (see Fig. 6), by setting the retarding potential $\phi_{\mathrm{S}}$ to zero, and using a set of measures of the upstream and downstream collected currents to deduce the Mach number (parallel drift speed normalized to the acoustic velocity). In order to complete the SOL plasma analysis, it would be interesting to mount a RFA and a Langmuir probe tip on the same head. Both temperatures could thus be measured simultaneously, the electron temperature from the Langmuir probe, and the ion temperature using the averaging procedure discussed in the previous paragraph and in Sec. IV.B. This would provide a value for the acoustic velocity and, by using the measure of the Mach number as discussed above, would yield the plasma drift velocity in dimensional units. 


\section{$\underline{\text { References }}$}

${ }^{1}$ P.C. Stangeby, G.M. McCracken, Nucl. Fusion 30, 1225 (1990).

${ }^{2}$ P.C. Stangeby, The plasma boundary of magnetic fusion devices (Institute of Physics

Publishing, London, 2000).

${ }^{3}$ G.F. Matthews, R. A. Pitts, G.M. McCracken, P.C. Stangeby, Nucl. Fusion 31, 1495 (1991).

${ }^{4}$ H. Weisen, M. Von Hellermann, A. Boileau, L.D. Horton, W. Mandl, H.P. Summers, Nucl. Fusion 29, 2187 (1989).

${ }^{5}$ M. Von Hellermann, W. Mandl, H.P. Summers, H. Weisen, A. Boileau, P.D. Morgan, H. Morsi, R. Koenig, M.F. Stamp, R. Wolf, Rev. Sci. Instrum. 61, 3479 (1990).

${ }^{6}$ M.F. Stamp, H.P. Summers, Proceedings of Contributed Papers, 17th European Conference on Controlled Fusion and Plasma Heating, Amsterdam, 1990, edited by G. Briffod, Adri Nijsen-Vis, F.C. Schüller (European Physical Society, 1990), Vol. 14B, part III, p. 1602.

${ }^{7}$ G.F. Matthews, J. Phys. D 17, 2243 (1984).

${ }^{8}$ A.S. Wan, T.F. Yang, B. Lipschultz, B. LaBombard, Rev. Sci. Instrum. 57, 1542 (1986).

${ }^{9}$ R.A. Pitts, Ion Energy, Sheath Potential and Secondary Electron Emission in the Tokamak Edge, PhD Thesis, University of London, 1991.

${ }^{10}$ R.A. Pitts, Phys. Fluids B 3, 2871 (1991).

${ }^{11}$ R.A. Pitts, Contrib. Plasma Phys. 36, 87 (1996).

${ }^{12}$ C.P. DeNeef, A.J. Theiss, Rev. Sci. Instrum. 50, 378 (1979).

${ }^{13}$ A.W. Molvik, Rev. Sci. Instrum. 52, 704 (1981).

${ }^{14}$ S.G. Ingram, N. St. J.Braithwaite, J. Appl. Phys. 68, 5519 (1990).

${ }^{15}$ C. Böhm, J. Perrin, Rev. Sci. Instrum. 64, 31 (1993).

${ }^{16}$ G.F. Matthews, Plasma Phys. Control. Fusion 36, 1595 (1994).

${ }^{17}$ C. Boucher, C.S. MacLatchy, G. Le Clair, J.L. Lachambre, M. St-Onge, J. Nucl. Mater. 176-177, 1050 (1990). 
${ }^{18}$ N. Tsois, C. Dorn, G. Kyriakakis, M. Markoulaki, M. Pflug, G. Schramm, P.

Theodoropoulos, P. Xantopoulos, M. Weinlich, et al., J. Nucl. Mater. 266-269, 1230 (1999).

${ }^{19}$ A. Hatayama, H. Segawa, R. Schneider, D.P. Coster, N. Hayashi, S. Sakurai, N. Asakura,

M. Ogasawara, Nucl. Fusion 40, 2009 (2000).

${ }^{20}$ S.K. Erents, A.V. Chankin, G.F. Matthews, P.C. Stangeby, Plasma Phys. Control. Fusion 42, 905 (2000).

${ }^{21}$ G.A. Emmert, R.M. Wieland, A.T. Mense, J.N. Davidson, Phys. Fluids 23, 803 (1980).

${ }^{22}$ R.C. Bissel, P.C. Johnson, Phys. Fluids 30, 779 (1987).

${ }^{23}$ P.C. Stangeby, Phys. Fluids 27, 2699 (1984).

${ }^{24}$ I.H. Hutchinson, Phys. Fluids 30, 3777 (1987).

${ }^{25}$ D. Poirier, C. Boucher, Proceedings of Contributed Papers, 25th European Conference on Controlled Fusion and Plasma Physics, Prague, 1998, edited by P. Pavlo (European Physical Society, Petit-Lancy, 1998), Vol. 22C, p. 1602.

${ }^{26}$ J.P. Gunn, C. Boucher, P. Devynck, I. Duran, K. Dyabilin, J. Horacek, M. Hron, J. Stöckel, G. Van Oost, H. van Goubergen, F. Zacek, Phys. Plasmas 8, 1995 (2001).

${ }^{27}$ K-S. Chung, I.H. Hutchinson, Phys. Rev. A 38, 4721 (1988).

${ }^{28}$ J.P. Gunn, Phys. Plasmas 8, 1040 (2001).

${ }^{29}$ I.H. Hutchinson, Phys. Fluids B 3, 847 (1991).

${ }^{30}$ C.K. Birdsall, A.B. Langdon, Plasma Physics via Computer Simulation (Institute of Physics Publishing, London, 1991).

${ }^{31}$ C.Z. Cheng, G. Knorr, J. Computational Phys. 22, 330 (1976).

${ }^{32}$ G. Manfredi, M. Shoucri, R. O. Dendy, A. Ghizzo, P. Bertrand, Phys. Plasmas 3, 202 (1996).

${ }^{33}$ E. Sonnendrücker, J. Roche, P. Bertrand, A. Ghizzo, J. Computational Phys. 149, 201 (1999). 
${ }^{34}$ F. Valsaque, G. Manfredi, J. Nucl. Mater. 290-293, 763 (2001).

${ }^{35} \mathrm{~F}$. Valsaque, G. Manfredi, Kinetic simulations of retarding field analyzer measurements in tokamak edge plasmas, Proceedings of Contributed Papers, 28th European Conference on Controlled Fusion and Plasma Physics, Funchal, 2001, to be published by European Physical Society.

${ }^{36}$ C. Boucher, L.-G. Thibault, J.P. Gunn, J.-Y. Pascal, P. Devynck, J. Nucl. Mater. 290-293, 561 (2001). 

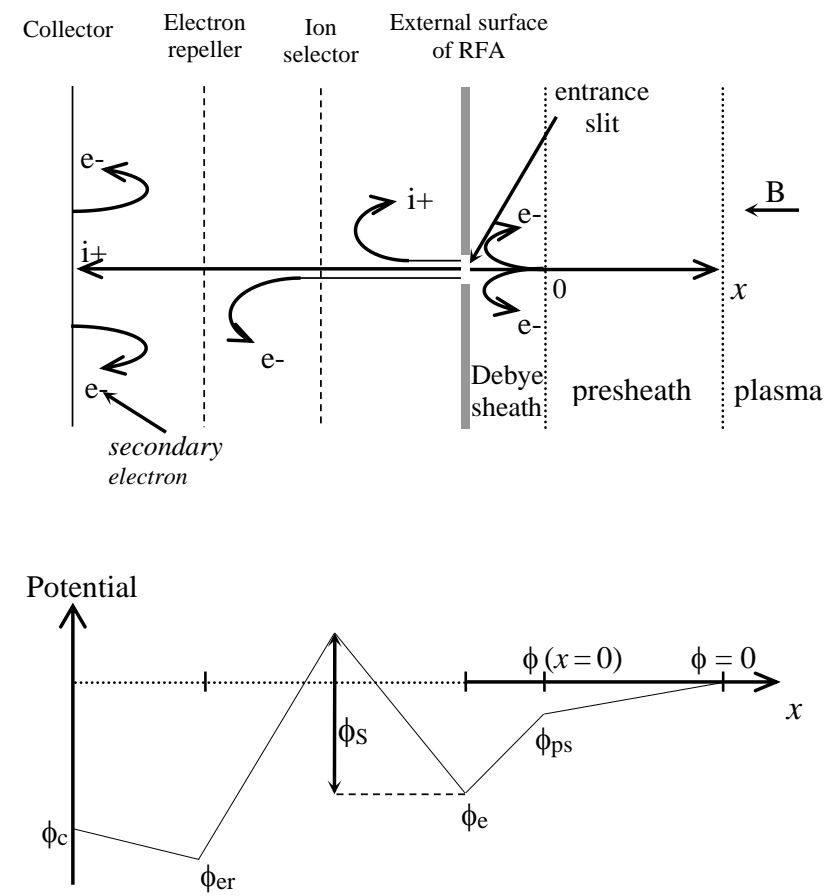

FIG. 1: Experimental device: schematic view of the Retarding Field Analyzer, on the upstream side.

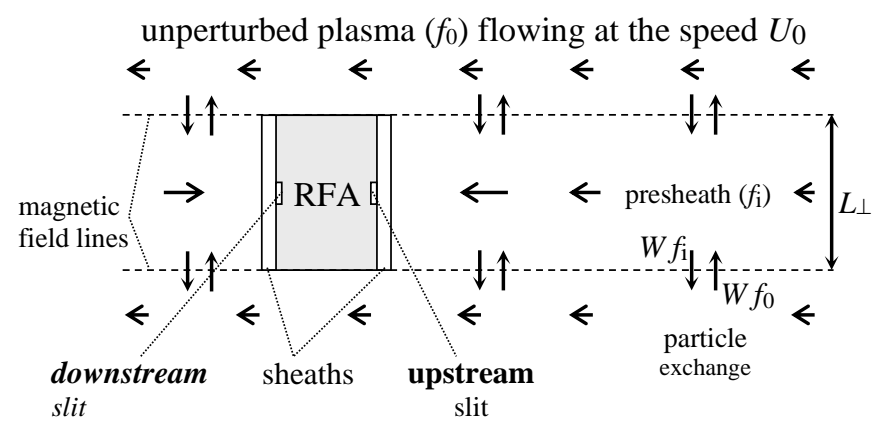

FIG. 2: RFA in a flowing plasma, in the presence of a strong magnetic field. 


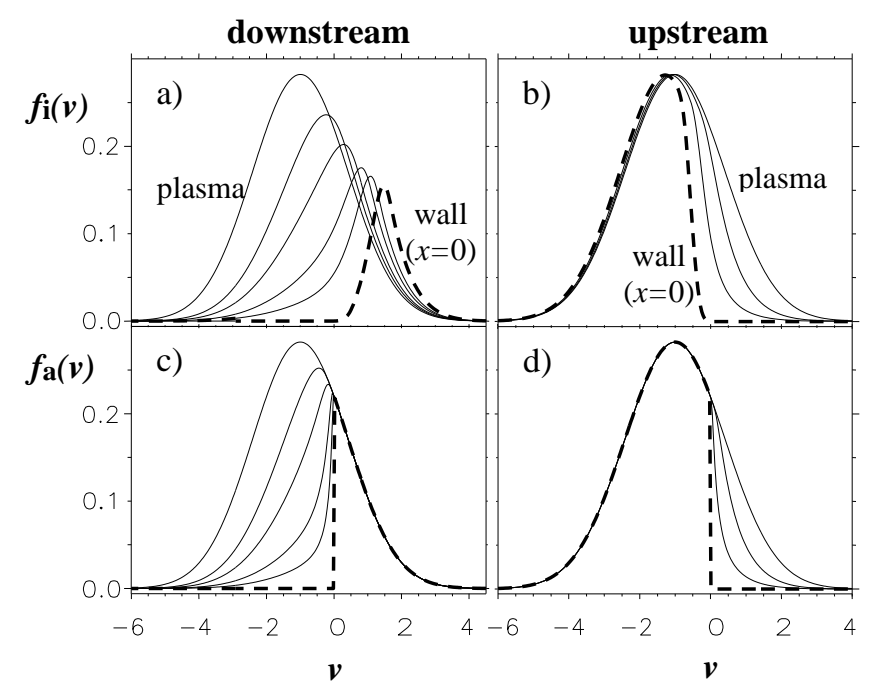

FIG. 3: a-b: Vlasov simulations; c-d: analytical model. Ion distribution profiles $(\tau=2$ and $U_{0}=1$ ) for different positions from the plasma to the wall (dashed curve). (Left: downstream side, $x=15,1.5,0.7,0.25,0.1$, and 0 ; right: upstream side, $x=0,0.15,0.65$, and 15 , positions normalized to $c_{\mathrm{e}} / W$.) 


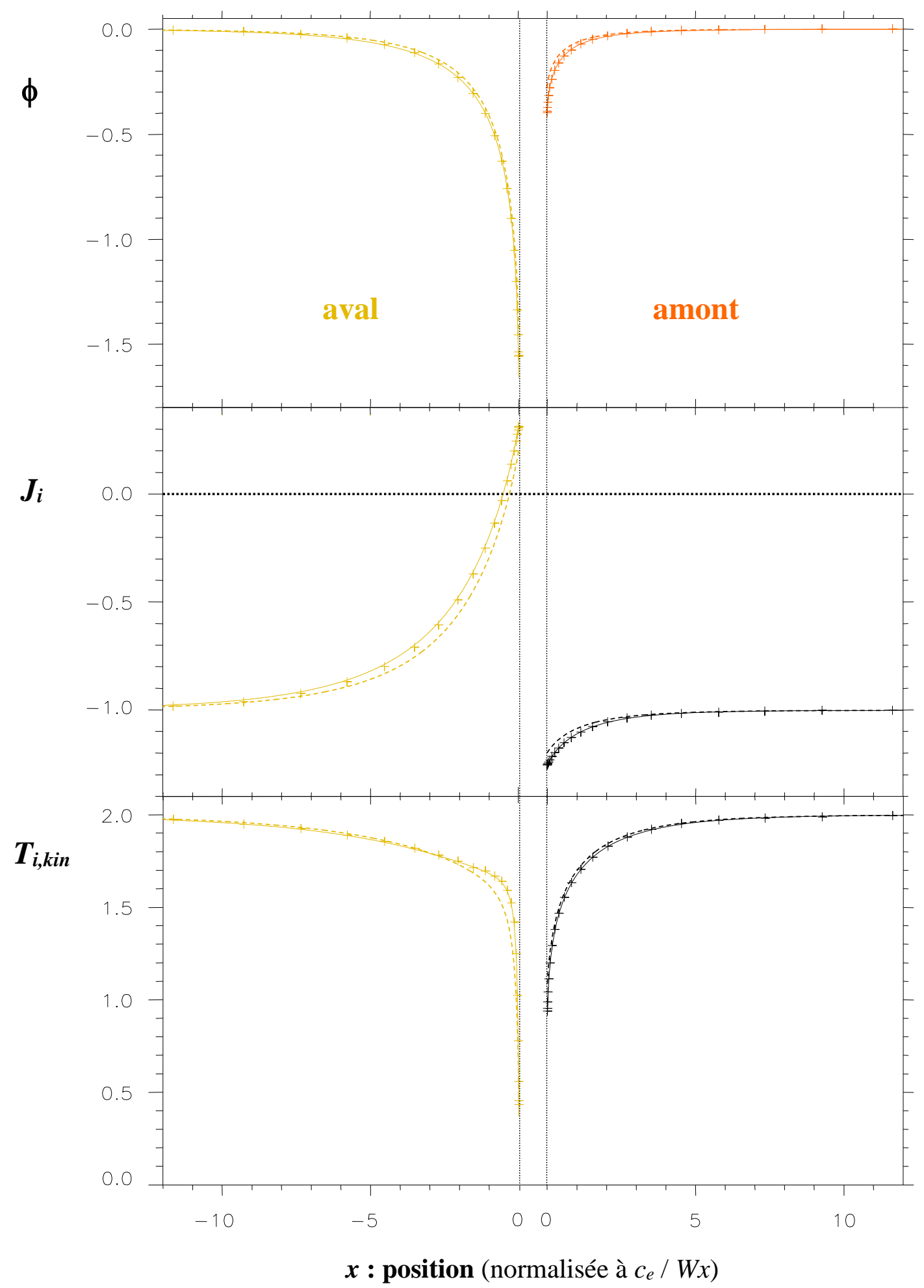

FIG. 4: Electric potential, ion current and ion kinetic temperature, respectively normalized to $k_{\mathrm{B}} T_{\mathrm{e}}, n_{0} c_{\mathrm{e}}$ and $T_{\mathrm{e}}$ (PIC: +, Vlasov: solid line, analytical model: dashed line), on each side of the RFA analyzer (for $\tau=2$ and $U_{0}=1$ ). 


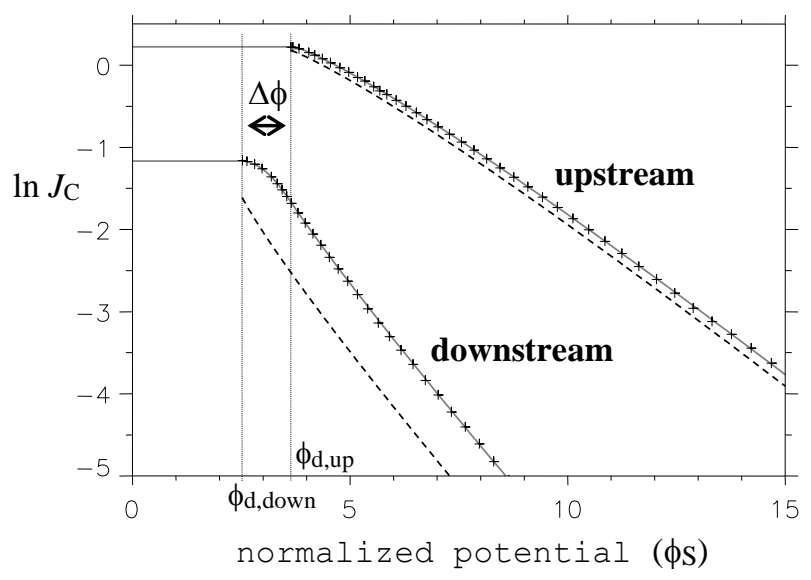

FIG. 5: PIC (+), Vlasov (solid line) and analytical model (dotted line) RFA characteristics. (Current normalized to $n_{0} c_{\mathrm{e}}, \tau=2$ and $U_{0}=1$ ).

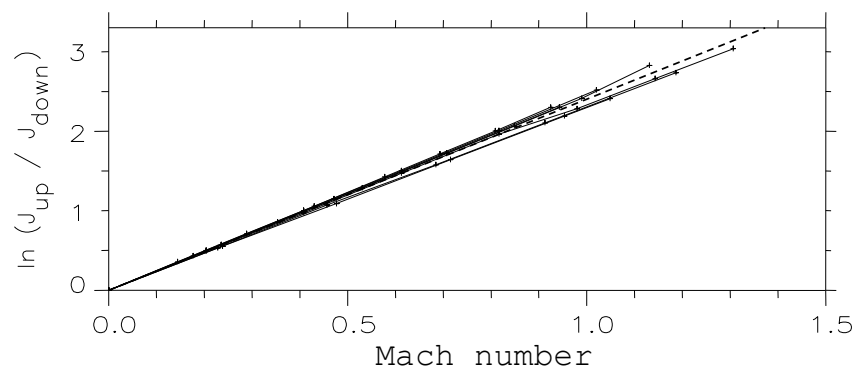

FIG. 6: Upstream-to-downstream current ratio (for $\phi_{\mathrm{S}}=0$ ) from Vlasov numerical simulations. $\tau=0.1,0.2,0.5,1,2,3.5$, and 5 . The dotted line is the kinetic model of Chung and Hutchinson. The Mach number is equal to the drift velocity normalized to $\mathrm{c}_{\mathrm{S}}$. 


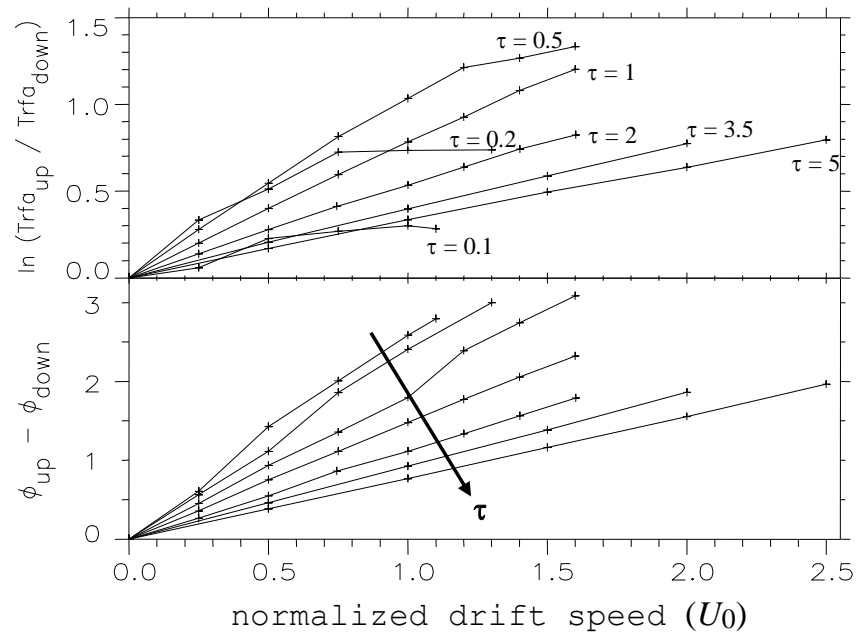

FIG. 7: Upstream-to-downstream RFA temperature ratio and difference potential $\Delta \phi$ from Vlasov numerical simulations. $\tau=0.1,0.2,0.5,1,2,3.5$, and 5 .

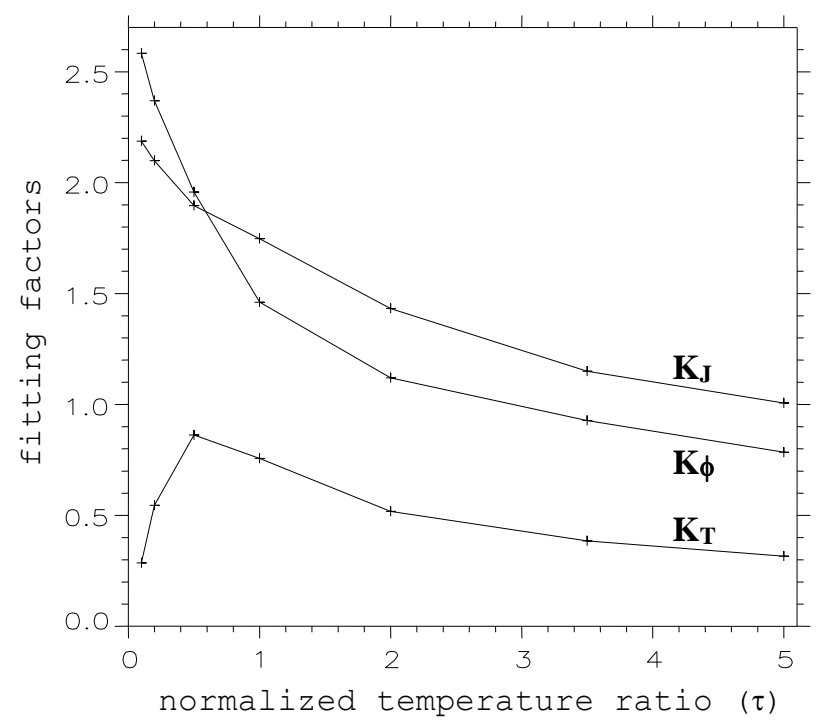

FIG. 8: Fitting factors $K_{\mathrm{J}}, K_{\mathrm{T}}$, and $K_{\phi}$ (expressed in unit of $\left.c_{\mathrm{e}}^{-1}\right)$ as a function of $\tau$. 


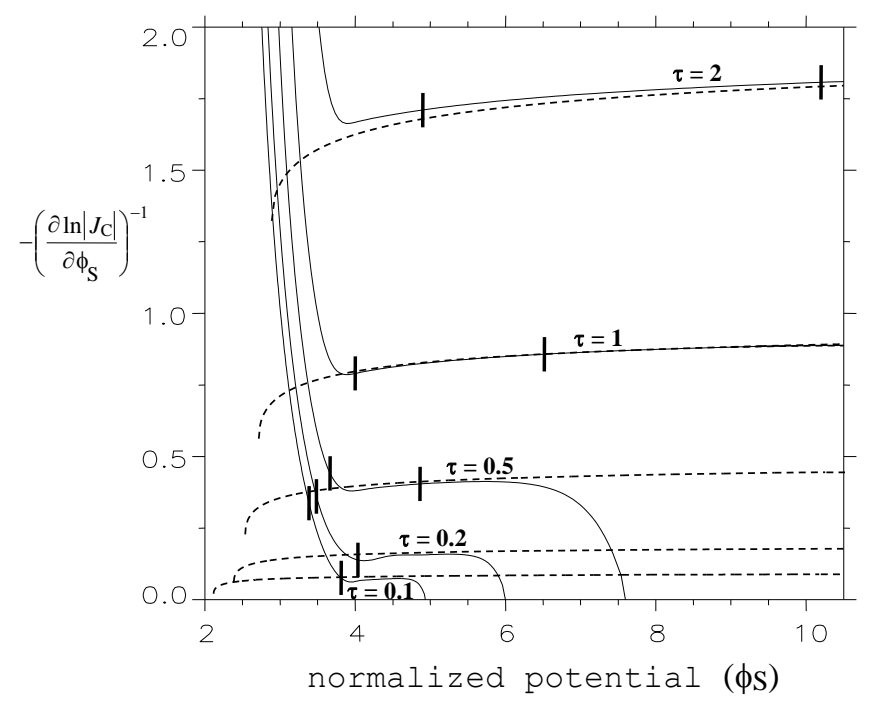

FIG. 9: Numerical (solid line) and analytical (dotted line) RFA temperature as a function of the retarding potential, on the downstream case for $U_{0}=0.5$ and $\tau=0.1,0.2,0.5,1$, and 2 . The thick vertical segments delimit the intervals where the RFA temperatures were estimated.

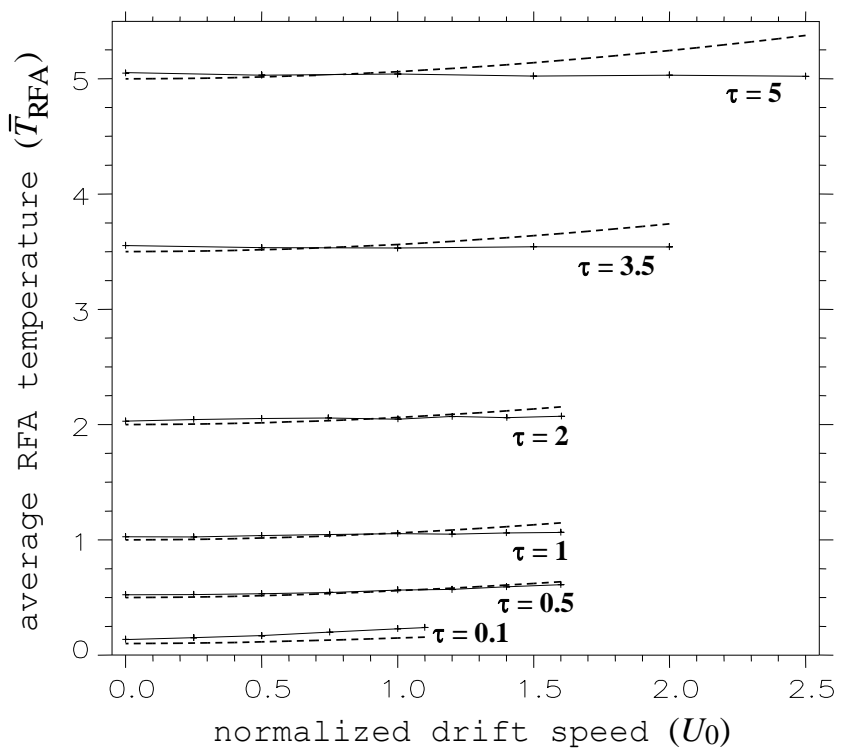

FIG. 10: Numerical (solid line) and analytical (dotted line) average RFA temperatures (normalized to $\mathrm{T}_{\mathrm{e}}$ ), for $\tau=0.1,0.5,1,2,3.5$, and 5 . 